\title{
(6) OPEN ACCESS \\ Relative deprivation in income and mortality by leading causes among older Japanese men and women: AGES cohort study
}

\author{
Naoki Kondo, ${ }^{1}$ Masashige Saito, ${ }^{2}$ Hiroyuki Hikichi, ${ }^{3}$ Jun Aida, ${ }^{4}$ Toshiyuki Ojima, ${ }^{5}$ \\ Katsunori Kondo, ${ }^{2,3}$ Ichiro Kawachi ${ }^{6}$
}

\begin{abstract}
- Additional material is published online only. To view please visit the journal online (http://dx.doi.org/10.1136/jech2014-205103).

${ }^{1}$ School of Public Health, the University of Tokyo, Tokyo, Japan

${ }^{2}$ Nihon Fukushi University, Nagoya, Japan

${ }^{3}$ Chiba University, Chiba, Japan

${ }^{4}$ Tohoku University, Sendai,

Japan

${ }^{5}$ Hamamatsu University School of Medicine, Hamamatsu, Japan

${ }^{6}$ Harvard School of Public Health, Boston, USA
\end{abstract}

\section{Correspondence to} Dr Naoki Kondo,

Floor 3, Medical Building \#3, School of Public Health, the University of Tokyo, 7-3-1 Hongo, Bunkyo-ku, Tokyo 113-0033, Japan; naoki-kondo@umin.ac.jp

Received 16 October 2014 Revised 22 January 2015 Accepted 31 January 2015 Published Online First 19 February 2015

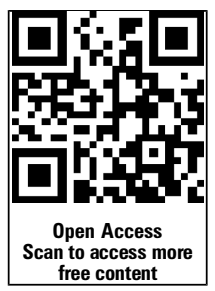

\section{ABSTRACT}

Background Relative deprivation of income is hypothesised to generate frustration and stress through upward social comparison with one's peers. If psychosocial stress is the mechanism, relative deprivation should be more strongly associated with specific health outcomes, such as cardiovascular disease (compared with other health outcomes, eg, non-tobacco-related cancer).

Methods We evaluated the association between relative income deprivation and mortality by leading causes, using a cohort of 21031 community-dwelling adults aged 65 years or older. A baseline mail-in survey was conducted in 2003. Information on cause-specific mortality was obtained from death certificates. Our relative deprivation measure was the Yitzhaki Index, derived from the aggregate income shortfall for each person, relative to individuals with higher incomes in that person's reference group. Reference groups were defined according to gender, age group and same municipality of residence.

Results We identified 1682 deaths during the 4.5 years of follow-up. A Cox regression demonstrated that, after controlling for demographic, health and socioeconomic factors including income, the HR for death from cardiovascular diseases per SD increase in relative deprivation was $1.50(95 \% \mathrm{Cl} 1.09$ to 2.08$)$ in men, whereas HRs for mortality by cancer and other diseases were close to the null value. Additional adjustment for depressive symptoms and health behaviours (eg, smoking and preventive care utilisation) attenuated the excess risks for mortality from cardiovascular disease by $9 \%$. Relative deprivation was not associated with mortality for women.

Conclusions The results partially support our hypothesised mechanism: relative deprivation increases health risks via psychosocial stress among men.

\section{BACKGROUND}

The association between income and health is well established, wherein income represents the power to purchase goods and services to maintain health. ${ }^{1}$ Beyond this direct relationship, researchers have posited the potential health impact of income relative to others in the social group. The impact of relative income on health can be explored through the contextual effects of income distribution on individual health, using a multilevel analytic strategy. $^{2} \quad 3$ A meta-analysis of multilevel studies revealed that an individual residing in a society with higher income inequality experiences increased risk of mortality, independent of individual socioeconomic status (including absolute income), compared with those living in a more equal society. ${ }^{45}$ An alternative approach to investigating the relative income hypothesis has focused on the individual-level association between a sense of 'relative deprivation' and health. Runciman argued that having less access to goods compared with others in a group (reference group) may lead to feelings of frustration, anxiety, unfairness and resentment, and these in turn may negatively affect health. In their meta-analysis of social psychological studies, Smith et $a l^{6}$ reported that relative deprivation has been linked to a range of social outcomes including riots, crime, lower educational attainment and poor health. In theory, relative deprivation can arise from social comparisons in different domains (such as possession of material goods), but the most frequent test of the hypothesis has been in the income domain. ${ }^{7}$

Epidemiological studies have suggested an association between relative income deprivation and various health outcomes, including stress-related health behaviour, such as smoking, ${ }^{8}$ mental health services utilisation, ${ }^{9}$ poor self-rated health, ${ }^{10-13}$ functional disability ${ }^{14}$ and mortality. ${ }^{15}$ However, to the best of our knowledge, no studies have investigated the differential impacts of relative deprivation on cause-specific mortality among older adults. ${ }^{16}$ If relative deprivation affects health through psychosocial stress-primarily via invidious social comparisons-then relative deprivation ought to be associated more strongly with mortality from diseases related to psychosocial stress. Therefore, the purpose of this study was to evaluate the association between relative income deprivation and the risk of mortality from major causes, using data from a large prospective cohort of Japanese older adults. Our secondary purpose was to evaluate the role of health behaviour and depressive symptoms as potential mediators in the pathway linking relative deprivation and cause-specific mortality. Japan was previously considered an egalitarian society; however, statistics from the past few decades have shown poverty rates to be continuously higher than the average of the member states of the Organization for Economic Cooperation and Development, due in part to a long stagnation of the economy and a rapidly ageing population. ${ }^{17}$ Moreover, as suggested by recent national representative data and systematic reviews, the country's structure of health disparity may not be typical. Specifically, poorer health and health behaviour 
among some socially privileged people, namely managers and professionals, has been shown. ${ }^{18}$

\section{METHODS}

Data

Data for this study came from the Aichi Gerontological Evaluation Study (AGES), a Japanese longitudinal panel study of older adults. The detailed protocol of AGES is available elsewhere. ${ }^{19}$ Briefly, the AGES baseline survey was carried out in 2003. Inclusion criteria for study participants were community-dwelling older adults, aged 65 years or older, residing in eight rural and suburban municipalities (out of 1976 Japanese municipalities at the time), and who were physically and cognitively independent. Functional status was ascertained from the official database of the public long-term care insurance system in Japan. Those who were identified as requiring assistance in using a toilet, eating meals and changing clothes were excluded from the baseline cohort. For eligible participants, we conducted a baseline mail-in survey $(n=59622$ individuals). We gathered information on baseline sociodemographic status, lifestyle factors, utilisation of healthcare services and social relationships. ${ }^{20}$ In total, 32891 participants responded to the survey invitation $(55.1 \%$ response, which is comparable to similar surveys of community-dwelling residents). In the analysis for the present study, we used data from 21031 individuals (9602 men and 11429 women) who were residents of municipalities where mortality registry microdata were available and successfully linked to the baseline data. The study protocol was approved by the Ethical Review Board of the Nihon Fukushi University (No. 10-05).

\section{Measurement}

Mortality data

The cause-of-death data provided by the national government were based on the death certificate that included the underlying causes of deaths, coded according to the International Classification of Diseases V.10 (ICD-10). For this study, we examined two major causes: malignant neoplasms or cancers (ICD-10 codes: C00-C97) and cardiovascular diseases (CVD; including heart and cerebrovascular diseases, ICD-9 codes: I01-I52, I60-I69). Owing to limited numbers, other less common causes were classified into an 'other' category. In addition, following Eibner and Evans, ${ }^{8}$ we created another cause-of-death category: tobacco-related cancers (cancers of the lip, oral cavity, pharynx, trachea, tracheal branch and lung: I4049 , I60-65), as tobacco smoking is partly a behavioural response to daily stress.

\section{Relative deprivation in income}

Following recent studies, ${ }^{16}$ we used the Yitzhaki Index to operationalise the construct of relative deprivation, which is derived from the formula:

$$
\text { YitzhakiIndex }_{i}=\frac{1}{N} \sum_{j}\left(y_{j}-y_{i}\right) I_{i j} \quad I_{i j}= \begin{cases}1, & \text { if } y_{i}<y_{j} \\ 0, & \text { if } y_{i} \geq y_{j}\end{cases}
$$

where the amount of relative deprivation for individual $i$ is the sum of the income shortfall compared with the income of all individuals $\mathrm{j}$, whose income exceeds individual i's. ${ }^{21}$ We divided that value by the number $(\mathrm{N})$ of people in i's reference group (in order to make the Index scale-invariant, ie, avoid the situation where larger reference groups spuriously result in greater Index values). When measuring relative deprivation, it is important to carefully consider the definition of reference groups (ie, the groups with which persons make social comparisons). ${ }^{22}$ Ishida reported that among Japanese men, satisfaction with salary was based on reference groups defined by demographic status, including age group and same area of residence, more than socioeconomic position (eg, occupational class and educational attainment). In contrast, this tendency was weak among women. ${ }^{23}$ Given this study result, we calculated the Yitzhaki Index based on multiple definitions of reference groups, using alternative combinations of basic demographic information, namely gender, age group and/or same municipality of residence.

For the Yitzhaki Index, household income was made proportional to the number of household members, by dividing the gross income by the square root of the number of people in the household. The income question included 14 categories, and the midpoints were set as the household income in each category.

\section{Covariates}

In terms of potential confounding factors, we considered demographic variables (age, gender and marital status), socioeconomic status (absolute income and educational attainment) and health status (disease and medical care utilisation). We also evaluated the contribution of depressive symptoms, measured using the validated Geriatric Depression Scale short version, and health behaviours (smoking, alcohol consumption, exercise habits and having health check-ups) as potential mediators linking relative deprivation to stress-related mortality. ${ }^{24} 25$

\section{Statistical analysis}

Imputation of missing values

AGES data included missing values. As the AGES questionnaire covered a wide range of variables, we used a multiple imputation technique through the Markov chain Monte Carlo method under the missing at random assumption. We created five imputed data sets based on different initial values. Using each data set, we first estimated the Kaplan-Meier survival curves and visually confirmed the proportionality assumption in mortality rates across levels of relative deprivation and demographic factors. Next, we ran Cox's proportional hazard regression controlling for potential confounding factors. After calculating unadjusted HRs and 95\% CIs, we created multivariable-adjusted models. To evaluate the role of potential mediators, we ran models further adjusting for health behaviours and depressive symptoms. Finally, we combined estimates based on five imputed data sets using the SAS PROC MIANLYZE procedure.

An issue when modelling both absolute income and relative income deprivation simultaneously is the strong correlation between the two variables. Potential collinearity due to the correlation could cause mixed findings in the association between relative deprivation and health. ${ }^{26}$ In this study, we sought to resolve this issue using stratified Cox regression, which is an approach to adjust a covariate, when the calculation of the covariate estimate is not required. ${ }^{27}$ We used the STRATA option SAS PROC PHREG procedure (SAS Institute Inc, Cary, North Carolina, USA) and used income quartiles as strata. Since the prevalence of stress-related behaviour (eg, smoking), relevant disease profiles and overall health risk due to psychosocial stress differs for males and females, all analyses were stratified by gender. $^{28}$ 
Sensitivity analysis

To evaluate the robustness of our findings, we further evaluated alternative results using the original data that were not multiply imputed (with up to $22.9 \%$ missing data for income). In addition, we conducted a stratified Cox regression using income quintiles rather than quartiles for the stratification variable.

\section{RESULTS}

During a maximum of 1666 days (4.5 years) of follow-up, we identified 1062 deaths during 35795 person-years of observation in men and 620 deaths during 42499 person-years in women. Descriptive analysis showed that overall, in both men and women, mortality from the three selected causes was positively associated with higher relative deprivation, lower household income, older age, unmarried status, lower educational attainment, currently needing medical care, currently smoking, no alcohol consumption, less walking, no recent health checkups and having depressive symptoms (table 1 and online supplementary table S1). The results of the Cox regression showed that mortality had a positive association with higher relative deprivation and lower absolute income. However, the association was statistically evident for mortality from CVD among men. For example, when relative deprivation was defined based on comparison with others of the same gender and residing in the same municipality, the crude HR for CVD mortality among men was 1.23 (95\% CI 1.09 to 1.38) for each SD unit increase in relative deprivation. The HR was 1.09 (95\% CI 0.99 to 1.21 ) for cancer mortality, and 1.19 (95\% CI 1.00 to 1.41) for all other causes of mortality. The corresponding HRs among women were 1.09 (0.93 to 1.27) for CVD, 1.10 (0.94 to 1.29) for cancer and 0.98 (0.82 to 1.19) for other causes (table 2). The HRs for mortality by tobacco-related cancers were 1.01 (0.83 to 1.23$)$.

After additional adjustment for socioeconomic status including absolute income, the adjusted HR of relative deprivation defined by gender and municipality increased to 1.54 (95\% CI 1.11 to 2.13 ) for CVD mortality, whereas they were 0.94 (95\% CI 0.74 to 1.19 ) for cancer and 1.00 (95\% CI 0.66 to 1.51 ) for other causes. Further adjustment for health behaviour and depressive symptoms (ie, potential mediators between relative deprivation and mortality) attenuated the association slightly. For example, the adjusted HR of relative deprivation defined by gender and municipality attenuated from 1.54 to 1.45 in men. Similar to the crude models, we did not find clear independent associations between relative deprivation and mortality among women (table 2; for full results, please see online supplementary table S2).

Sensitivity analysis using non-imputed data showed similar results with slightly smaller HRs. The adjusted HR of relative deprivation, defined by gender and municipalities adjusted for socioeconomic factors, was 1.36 (95\% CI 1.06 to 1.75 ), which was attenuated to 1.24 (95\% CI 0.94 to 1.62 ) in the model additionally adjusted for health behaviour and depressive symptoms (see online supplementary table S3). The results of further sensitivity analyses using income quintile as the stratification variable did not substantially change the results of our original analysis (see online supplementary table S4).

\section{DISCUSSION}

The key findings of this study are twofold. First, among older Japanese men, relative deprivation may be associated with increased risk for mortality from CVD (but not cancer or other causes), independent of absolute income; no association was observed among women. Second, potential mediators, including health behaviours and depressive symptoms, partially explained the association between relative deprivation and mortality from CVD. Nevertheless, relative deprivation was not independently associated with mortality from tobacco-related cancers.

To the best of our knowledge, there has been one previous report evaluating the association between Yitzhaki-based relative deprivation and mortality from specific causes. Using data from working-age men from the US National Health Interview Survey, Eibner and Evans found that relative deprivation was associated with mortality from coronary heart disease $(48 \%$ increase in risk for each SD unit increase in the Yitzhaki Index) and tobacco-related cancers (58\% increase). In contrast, the associations were less clear for mortality from all other cancers, accidents and adverse events. ${ }^{8}$ Our findings (based on older population data) suggest a comparable magnitude of association between relative deprivation and coronary heart disease among men to that of Eibner and Evans. Thus, the hypothesis that social comparisons may induce psychosocial stress, which increases the risk for CVD (at least among men), has been supported. Although Eibner and Evans also identified an increased risk for tobacco-related cancers among relatively deprived working-age men, this was not found in our study. A possible explanation for this discrepancy may relate to age differences. Younger people may be more likely to select 'starting tobacco' as their response to behaviour-change triggers, including psychosocial stress, compared with older people whose lifestyles are largely established. In addition, although CVD risk could increase due to both chronic vascular damage and acute events triggered by stressful experiences, cancer risks can only be increased chronically. Therefore, since tobacco-related cancer requires a few decades to develop, our follow-up period of 4 years might not have been long enough to capture the aetiological association between relative deprivation, stress, smoking and cancer.

In our analysis, additional adjustment for health behaviours and depressive symptoms attenuated the HRs for CVD mortality. Although this was in line with our a priori hypothesis, the degree of attenuation was modest (mortality risk per SD unit increase in relative deprivation, defined by gender and municipality, attenuated by nine basis points from $54 \%$ to $45 \%$; table 2). One explanation for this finding is that the induction period between exposure and mortality varies for relative deprivation and health behaviours. For example, cancer mortality is likely to reflect behaviours that were adopted decades prior to the observation period, thereby introducing measurement error in our mediation analysis.

Among women, we did not find any association between relative deprivation and mortality. This is consistent with our previous findings on the association between relative deprivation and risk of functional decline. ${ }^{14}$ We note that absolute income itself was not associated with mortality by leading causes among women (table 2). The weaker association between income and mortality among women (compared with men) has been noted in previous studies based in Japan. ${ }^{29}$ It thus appears that household income as an indicator of socioeconomic position has a different meaning for Japanese women compared with men. This may be explained as follows: among older Japanese, the most prevalent occupation for women is 'home-maker'; thus, older Japanese women may not be psychologically primed to make income comparisons in the same manner as wage earners.

Our study has several strengths. First, this is the first study to evaluate the association between relative income deprivation and risk for mortality by leading causes among older men and 
Table 1 Cause-specific mortality rates (per 1000 people per year) by relative deprivation and household income ( $\mathrm{N}=21$ 031): AGES cohort, 2003-2007

\begin{tabular}{|c|c|c|c|c|c|c|c|c|c|c|}
\hline & \multicolumn{5}{|c|}{ Men ( $N=9602)$} & \multicolumn{5}{|c|}{ Women $(\mathrm{N}=11$ 429) } \\
\hline & $\mathrm{N}$ & Cancer & CVD & Other & $\begin{array}{l}\text { Tobacco } \\
\text { cancer }\end{array}$ & $\mathbf{n}$ & Cancer & CVD & Other & $\begin{array}{l}\text { Tobacco } \\
\text { cancer }\end{array}$ \\
\hline \multicolumn{11}{|c|}{ Relative deprivation by definitions of reference groups, quartile (10 000 yen) } \\
\hline \multicolumn{11}{|l|}{ Gender and municipality of residence } \\
\hline Q1 (men 0-30, women 0-30) & 2400 & 10.8 & 5.8 & 3.3 & 3.1 & 2902 & 4.3 & 4.5 & 3.2 & 0.8 \\
\hline Q2 (men 30-60, women 30-66) & 2393 & 12.6 & 7.2 & 3.8 & 3.8 & 2820 & 4.5 & 4.1 & 3.0 & 0.9 \\
\hline Q3 (men 60-108, women 66-114) & 2390 & 11.9 & 7.7 & 3.5 & 2.8 & 2873 & 5.7 & 4.4 & 2.6 & 1.0 \\
\hline Q4 (men 108+, women 114+) & 2419 & 14.2 & 9.2 & 5.5 & 3.6 & 2834 & 5.4 & 5.4 & 2.9 & 0.5 \\
\hline \multicolumn{11}{|l|}{ Gender and age group } \\
\hline Q1 (men 0-27, women 0-32) & 2405 & 11.0 & 5.8 & 3.2 & 3.5 & 2980 & 4.4 & 4.4 & 3.0 & 0.8 \\
\hline Q2 (men 27-63, women 32-63) & 2338 & 12.3 & 7.4 & 3.9 & 3.4 & 2829 & 4.3 & 3.9 & 3.1 & 0.9 \\
\hline Q3 (men 63-109, women 63-115) & 2515 & 12.3 & 8.1 & 3.9 & 3.1 & 2742 & 5.9 & 4.3 & 2.4 & 0.8 \\
\hline Q4 (men 109+, women 115+) & 2344 & 14.1 & 8.7 & 5.1 & 3.5 & 2878 & 5.3 & 5.8 & 3.1 & 0.6 \\
\hline \multicolumn{11}{|l|}{ Gender, age group, municipality } \\
\hline Q1 (men 0-30, women 0-30) & 2399 & 11.0 & 6.0 & 3.4 & 3.5 & 2883 & 4.6 & 4.4 & 3.0 & 0.8 \\
\hline Q2 (men 30-60, women 30-64) & 2399 & 12.4 & 6.9 & 3.7 & 3.4 & 2805 & 4.2 & 4.1 & 3.0 & 1.0 \\
\hline Q3 (men 60-108, women 64-113) & 2424 & 12.9 & 8.1 & 3.6 & 3.1 & 2892 & 5.7 & 4.5 & 2.7 & 0.9 \\
\hline Q4 (men 108+, women 113+) & 2380 & 13.3 & 8.7 & 5.4 & 3.4 & 2849 & 5.4 & 5.4 & 2.8 & 0.5 \\
\hline \multicolumn{11}{|c|}{ Equivalised household income, quartile (10 000 yen) } \\
\hline Q1 (men 0-138, women 0-112) & 2378 & 14.6 & 9.0 & 5.1 & 3.5 & 2900 & 5.5 & 5.8 & 3.1 & 0.6 \\
\hline Q2 (men 138-202, women 112-195) & 2441 & 12.0 & 7.7 & 3.9 & 3.1 & 3234 & 5.5 & 4.1 & 2.3 & 0.8 \\
\hline Q3 (men 202-318, women 195-275) & 2301 & 12.6 & 7.8 & 4.0 & 3.2 & 2196 & 4.3 & 4.2 & 3.0 & 1.0 \\
\hline Q4 (men 318+, women 275+) & 2482 & 10.7 & 5.5 & 3.1 & 3.5 & 3100 & 4.5 & 4.4 & 3.2 & 0.8 \\
\hline
\end{tabular}

Tobacco cancer, tobacco-related cancers (cancers of the lip, oral cavity, pharynx, trachea, tracheal branch and lung).

AGES, Aichi Gerontological Evaluation Study; CVD, cardiovascular diseases.

women in a developed country. Second, we utilised a large population-based cohort, with objective and reliable mortality information, as well as statistical techniques to address missing information. Third, our sensitivity analyses confirmed the robustness of this study's findings. Nonetheless, some caution should be noted in interpreting the results of this study. Owing to the limited number of mortality cases, we were not able to evaluate the association between relative deprivation and

Table 2 HRs (95\% Cls) for cause-specific mortality by relative deprivation for three reference groups: AGES cohort data 2003-2007

\begin{tabular}{|c|c|c|c|c|c|}
\hline \multirow{2}{*}{$\begin{array}{l}\text { Relative deprivation } \\
\text { defined by }\end{array}$} & \multirow[b]{2}{*}{ Variables adjusted for } & \multicolumn{3}{|l|}{ Cause of death } & \multirow{2}{*}{$\begin{array}{l}\text { Tobacco-related } \\
\text { cancer }\end{array}$} \\
\hline & & Cancer & CVD & Other & \\
\hline \multicolumn{6}{|l|}{ Men } \\
\hline $\begin{array}{l}\text { Gender and municipality of } \\
\text { residence }\end{array}$ & $\begin{array}{l}\text { Crude } \\
\text { Demographic+SES } \\
+ \text { Health behaviour and depressive symptoms }\end{array}$ & $\begin{array}{l}1.09(0.99 \text { to } 1.21) \\
0.94(0.74 \text { to } 1.19) \\
0.90(0.72 \text { to } 1.14)\end{array}$ & $\begin{array}{l}1.23(1.09 \text { to } 1.38) \\
1.54(1.11 \text { to } 2.13) \\
1.45(1.04 \text { to } 2.03)\end{array}$ & $\begin{array}{l}1.19(1.00 \text { to } 1.40) \\
1.00(0.66 \text { to } 1.51) \\
0.94(0.62 \text { to } 1.42)\end{array}$ & $\begin{array}{l}1.01(0.83 \text { to } 1.23) \\
0.95(0.59 \text { to } 1.51) \\
0.92(0.57 \text { to } 1.48)\end{array}$ \\
\hline Gender and age group & $\begin{array}{l}\text { Crude } \\
\text { Demographic+SES } \\
\text { +Health behaviour and depressive symptoms }\end{array}$ & $\begin{array}{l}1.08(0.97 \text { to } 1.20) \\
0.91(0.69 \text { to } 1.20) \\
0.89(0.67 \text { to } 1.17)\end{array}$ & $\begin{array}{l}1.19(1.06 \text { to } 1.34) \\
1.60(1.03 \text { to } 2.48) \\
1.54(0.98 \text { to } 2.42)\end{array}$ & $\begin{array}{l}1.15(0.96 \text { to } 1.38) \\
1.04(0.63 \text { to } 1.73) \\
0.94(0.62 \text { to } 1.42)\end{array}$ & $\begin{array}{l}0.99(0.80 \text { to } 1.23) \\
0.91(0.52 \text { to } 1.58) \\
0.91(0.52 \text { to } 1.61)\end{array}$ \\
\hline $\begin{array}{l}\text { Gender, age group and } \\
\text { municipality of residence }\end{array}$ & $\begin{array}{l}\text { Crude } \\
\text { Demographic+SES } \\
\text { +Health behaviour and depressive symptoms }\end{array}$ & $\begin{array}{l}1.07(0.96 \text { to } 1.18) \\
0.92(0.73 \text { to } 1.17) \\
0.89(0.70 \text { to } 1.12)\end{array}$ & $\begin{array}{l}1.19(1.06 \text { to } 1.34) \\
1.50(1.09 \text { to } 2.08) \\
1.42(1.02 \text { to } 1.98)\end{array}$ & $\begin{array}{l}1.14(0.96 \text { to } 1.36) \\
1.02(0.67 \text { to } 1.53) \\
0.95(0.63 \text { to } 1.44)\end{array}$ & $\begin{array}{l}0.99(0.81 \text { to } 1.21) \\
0.90(0.56 \text { to } 1.45) \\
0.87(0.54 \text { to } 1.42)\end{array}$ \\
\hline \multicolumn{6}{|l|}{ Women } \\
\hline $\begin{array}{l}\text { Gender and municipality of } \\
\text { residence }\end{array}$ & $\begin{array}{l}\text { Crude } \\
\text { Demographic+SES } \\
+ \text { Health behaviour and depressive symptoms }\end{array}$ & $\begin{array}{l}1.10(0.94 \text { to } 1.29) \\
1.04(0.71 \text { to } 1.53) \\
1.00(0.68 \text { to } 1.48)\end{array}$ & $\begin{array}{l}1.09(0.93 \text { to } 1.27) \\
0.76(0.50 \text { to } 1.15) \\
0.74(0.48 \text { to } 1.12)\end{array}$ & $\begin{array}{l}0.98(0.82 \text { to } 1.19) \\
0.93(0.55 \text { to } 1.58) \\
0.90(0.54 \text { to } 1.52)\end{array}$ & $\begin{array}{l}0.85(0.55 \text { to } 1.31) \\
0.70(0.23 \text { to } 2.18) \\
0.71(0.23 \text { to } 2.18)\end{array}$ \\
\hline Gender and age group & $\begin{array}{l}\text { Crude } \\
\text { Demographic+SES } \\
+ \text { Health behaviour and depressive symptoms }\end{array}$ & $\begin{array}{l}1.10(0.94 \text { to } 1.29) \\
1.10(0.66 \text { to } 1.83) \\
1.05(0.63 \text { to } 1.77)\end{array}$ & $\begin{array}{l}1.09(0.93 \text { to } 1.28) \\
0.69(0.38 \text { to } 1.23) \\
0.66(0.36 \text { to } 1.20)\end{array}$ & $\begin{array}{l}1.00(0.83 \text { to } 1.21) \\
1.04(0.48 \text { to } 2.22) \\
0.98(0.46 \text { to } 2.08)\end{array}$ & $\begin{array}{l}0.87(0.57 \text { to } 1.32) \\
0.84(0.19 \text { to } 3.70) \\
0.88(0.20 \text { to } 3.88)\end{array}$ \\
\hline $\begin{array}{l}\text { Gender, age group and } \\
\text { municipality of residence }\end{array}$ & $\begin{array}{l}\text { Crude } \\
\text { Demographic+SES } \\
+ \text { Health behaviour and depressive symptoms }\end{array}$ & $\begin{array}{l}1.09(0.93 \text { to } 1.28) \\
1.01(0.69 \text { to } 1.48) \\
0.97(0.66 \text { to } 1.44)\end{array}$ & $\begin{array}{l}1.09(0.93 \text { to } 1.28) \\
0.77(0.51 \text { to } 1.16) \\
0.75(0.50 \text { to } 1.14)\end{array}$ & $\begin{array}{l}0.99(0.82 \text { to } 1.19) \\
0.97(0.58 \text { to } 1.61) \\
0.94(0.56 \text { to } 1.55)\end{array}$ & $\begin{array}{l}0.84(0.54 \text { to } 1.30) \\
0.64(0.20 \text { to } 1.98) \\
0.64(0.21 \text { to } 1.98)\end{array}$ \\
\hline
\end{tabular}


mortality by rarer causes, including suicide. Suicide may be more directly associated with psychosocial stress than other causes. This should be evaluated in future research, using a longer follow-up and a larger sample size. Moreover, missing data and attrition during follow-up may have introduced bias. Although we used a stratified Cox regression to adjust for absolute income, there might have been residual confounding within each stratum of absolute income. However, our sensitivity analysis using quintile points for stratification showed mostly the same HRs, suggesting that the extent of residual confounding was small. In addition, income may not be the socioeconomic indicator most used by older Japanese to compare themselves with others. The majority of older Japanese are out of the labour force, and thus may rely more on savings and other assets rather than income. Further studies should explore relative deprivation with regard to other socioeconomic indicators, including wealth and actual material conditions.

In conclusion, invidious social comparisons may increase the risk for mortality by CVD, but not by major cancers and other diseases in Japanese older men. The results of our study provide partial support for the relative deprivation hypothesis. More studies with detailed information on causes of death (cancers related to health behaviours, suicide, etc) may be required to gain a better understanding of the role of social comparisons on health. ${ }^{16}$ Moreover, those effects may be strongly moderated by broader social contexts, although evidence from other parts of the world is mostly lacking. ${ }^{8}$ Further studies in other settings, especially in developing countries, appear to be warranted. ${ }^{30}$

\section{What is already known on this topic}

- Relative deprivation of income may increase the risk for premature death by increasing psychosocial stress due to invidious social comparison.

- No study exists on the association between relative deprivation and mortality by stress-related diseases (cardiovascular diseases) and other causes less associated with psychosocial stress among older adults.

\section{What this study adds}

- Among older men, relative deprivation in income was associated with mortality by cardiovascular disease, but not from cancer and other causes.

- This association was not observed among women.

\begin{abstract}
Acknowledgements The authors thank other JAGES group members. Members of the JAGES group are as follows: KK (lead investigator), Hanazato $M, H H$, Miyaguni Y, Sasaki Y, Nagamine Y, Chiba University, Chiba; Ashida T, NK, Takagi D, Tani Y, The University of Tokyo, Tokyo; JA, Osaka K, Tsuboya T, Tohoku University, Miyagi; Jeong S, Murata C, Saito T, National Center for Geriatrics and Gerontology, Aichi; TO, Okada E, Hamamatsu University School of Medicine, Shizuoka; Shirai K, Todoriki $\mathrm{H}$, University of the Ryukyus, Okinawa; MS, Nihon Fukushi University, Aichi; Hirai $\mathrm{H}_{\text {, }}$ Iwate University, Iwate; Misawa J, Rikkyo University, Tokyo; Suzuki K, Aichi Gakuin University, Aichi; Ichida Y, Doctoral Institute for Evidence Based Policy, Tokyo; Takeda T, Seijoh University, Aichi; Yamamoto T, Kanagawa Dental University, Kanagawa; Nakade M, Tokaigakuen University, Aichi; Cable N, University College London, London; Tamakoshi A, Hokkaido University Graduate School of Medicine, Hokkaido; Fujino Y, University of Occupational and Environmental Health, Fukuoka;
\end{abstract}

Shobugawa Y, Niigata University, Niigata; Hayashi T, Tokai College of Medical Science, Aichi.

Contributors NK was responsible for the study conception, design, analysis, data interpretation and drafting the article. MS, $\mathrm{HH}$ and JA intensively revised the manuscript. IK supported the conception of the study and intensively revised the manuscript. TO and KK acquired the data and intensively revised the manuscript.

Funding This study used data from the Aichi Gerontological Evaluation Study (AGES), conducted by the Center for Well-being and Society, Nihon Fukushi University as one of its research projects. This study was supported in part by the MEXT-Supported Program for the Strategic Research Foundation at Private Universities, 2009-2013 and Grant-in-Aid for Scientific Research (23243070). NK was supported by the Ministry of Education, Culture, Sports, Science and Technology (MEXT), Japan (Nos. 21119002, 25253052) and the Ministry of Health, Labour and Welfare, Japan (H26-chouju-ippan-006)

Competing interests NK, MS, HH, JA, TO and KK had support from the Ministry of Education, Culture, Sports, Science and Technology (MEXT), Japan and the Ministry of Health, Labour and Welfare, Japan for the submitted work.

Ethics approval Ethical permission (No. 10-05) was provided by the Ethics Board at Nihon Fukushi University.

Provenance and peer review Not commissioned; externally peer reviewed.

Open Access This is an Open Access article distributed in accordance with the Creative Commons Attribution Non Commercial (CC BY-NC 4.0) license, which permits others to distribute, remix, adapt, build upon this work non-commercially, and license their derivative works on different terms, provided the original work is properly cited and the use is non-commercial. See: http://creativecommons.org/ licenses/by-nc/4.0/

\section{REFERENCES}

1 Kawachi I, Adler N, Dow W. Money, schooling, and health: mechanisms and causal evidence. Ann N Y Acad Sci 2010;1186:56-68.

2 Wilkinson RG, Pickett KE. Income inequality and population health: a review and explanation of the evidence. Soc Sci Med 2006;62:1768-84.

3 Subramanian SV, Kawachi I. Income inequality and health: what have we learned so far? Epidemiol Rev 2004;26:78-91.

4 Kondo N, van Dam RM, Sembajwe G, et al. Income inequality and health: the roles of population size, inequality threshold, period effects, and lag effects. J Epidemiol Community Health 2011;66:e11.

5 Kondo N, Sembajwe G, Kawachi I, et al. Income inequality, mortality, and self rated health: meta-analysis of multilevel studies. BMJ 2009;339:b4471.

6 Smith HJ, Pettigrew TF, Pippin GM, et al. Relative deprivation: a theoretical and meta-analytic review. Pers Soc Psychol Rev 2012;16:203-32.

7 Runciman WG. Relative deprivation and social justice: a study of attitudes to social inequality in twentieth-century. England: University of California Press, 1966.

8 Eibner C, Evans WN. Relative deprivation, poor health habits, and mortality. J Hum Resour 2005;40:591-620.

9 Eibner C, Sturn R, Gresenz CR. Does relative deprivation predict the need for mental health services? J Ment Health Policy Econ 2004;7:167-75.

10 Subramanyam M, Kawachi I, Berkman L, et al. Relative deprivation in income and self-rated health in the United States. Soc Sci Med 2009;69:327-34.

11 Kondo N, Kawachi I, Yamagata Z. Relative deprivation and perceived health among Japanese working-age population: exploring psychological pathways in income inequality and health. 41st Annual Meeting of Socierty for Epidemiologic Research, USA. Chicago, IL, USA, 2008.

12 Kondo N, Kawachi I, Subramanian SV, et al. Do social comparisons explain the association between income inequality and health? Relative deprivation and perceived health among male and female Japanese individuals. Soc Sci Med 2008;67:982-7.

13 Åberg Yngwe M, Fritzell J, Lundberg 0, et al. Exploring relative deprivation: is social comparison a mechanism in the relation between income and health? Soc Sci Med 2003:57:1463-73.

14 Kondo N, Kawachi I, Hirai $\mathrm{H}$, et al. Relative deprivation and incident functional disability among older Japanese women and men: prospective cohort study. J Epidemiol Community Health 2009;63:461-7.

15 Åberg Yngwe M, Kondo N, Hägg S, et al. Relative deprivation and mortalitylongitudinal study in a Swedish population of 4,7 million, 1990-2006. BMC Public Health 2012;12:664.

16 Adjaye-Gbewonyo K, Kawachi I. Use of the Yitzhaki Index as a test of relative deprivation for health outcomes: a review of recent literature. Soc Sci Med 2012;75:129-37

17 OECD. Crisis squeezes income and puts pressure on inequality and poverty: Organization for Economic Cooperation and Development 2013. http://www.oecd. org/els/soc/OECD2013-Inequality-and-Poverty-8p.pdf Accessed on. 
18 Wada K, Kondo N, Gilmour S, et al. Trends in cause specific mortality across occupations in Japanese men of working age during period of economic stagnation, 1980-2005: retrospective cohort study. BMJ 2012;344:e1191.

19 Nishi A, Kondo K, Hirai H, et al. Cohort profile: the ages 2003 cohort study in Aichi, Japan. J Epidemiol 2011;21:151-7.

20 Kondo K, ed. Health inequalities in Japan: an empirical study of the older people. Melbourne: Trans Pacific Press, 2010.

21 Yitzhaki S. Relative deprivation and the Gini coefficient. Q J Econ 1979:93:321-4.

22 Festinger L. A theory of social comparison processes. Hum Relations 1954;7:117-40.

23 Ishida J. A quantitative model of relative deprivation and reference group: an application of Yitzhaki's individual relative deprivation index. Sociol Theory Methods 2011;26:371-88.

24 Sugishita M, Asada T. Development of Geriatric Depression Scale short version. Jpn J Cogn Neurosci 2009;11:87-90.
25 Sheikh J, Yesavage J. Geriatic Depression Scale (GDS). Recent evidence and development of a shorter version. Clin Gerontol 1986;5:165-73.

26 Gravelle $\mathrm{H}$, Sutton M. Income, relative income, and self-reported health in Britain 1979-2000. Health Econ 2009;18:125-45.

27 Kalbfleisch JD, Prentice RL. The statistical analysis of failure time data. New York: John Wiley \& Sons, 1980.

28 Saito M, Kondo N, Kondo K, et al. Gender differences on the impacts of social exclusion on mortality among older Japanese: AGES cohort study. Soc Sci Med 2012;75:940-5

29 Kagamimori S, Gaina A, Nasermoaddeli A. Socioeconomic status and health in the Japanese population. Soc Sci Med 2009;68:2152-60.

30 Salti N. Relative deprivation and mortality in South Africa. Soc Sci Med $2010 ; 70: 720-8$ 